\title{
Cbl-b deficiency provides protection against UVB-induced skin damage by modulating inflammatory gene signature
}

\author{
Tej Pratap Singh ${ }^{1,2,4}$, Pablo A. Vieyra-Garcia ${ }^{1,2}$, Karin Wagner ${ }^{2}$, Josef Penninger ${ }^{3}$ and Peter Wolf ${ }^{1}$
}

\begin{abstract}
Exposure of skin to ultraviolet (UV) radiation induces DNA damage, inflammation, and immune suppression that ultimately lead to skin cancer. However, some of the pathways that regulate these events are poorly understood. We exposed mice to UVB to study its early effects in the absence of Cbl-b, a known suppressor of antitumor immune response in the skin. Cbl- $b^{-/-}$mice were protected from UV-induced cell damage as shown by the lower number of cyclobutane pyrimidine dimers and sunburn cells in exposed skin compared to wild-type mice. Microarray data revealed that deficiency of $\mathrm{Cbl}-\mathrm{b}$ resulted in differential expression of genes involved in apoptosis evasion, tumor suppression and cell survival in UV-exposed skin. After UVB, Cbl-b ${ }^{-1-}$ mice upregulated gene expression pattern associated with regulation of epidermal cell proliferation linked to Wnt signaling mediators and enzymes that relate to cell removal and tissue remodeling like MMP12. Additionally, the skin of $\mathrm{Cbl}_{-1} \mathrm{~b}^{-/-}$mice was protected from chronic inflammatory responses and epidermal hyperplasia in a 4-weeks UVB treatment protocol. Overall, our results suggest a novel role for Cbl-b in regulating inflammation and physiologic clearance of damaged cells in response to UVB by modulating inflammatory gene signature.
\end{abstract}

\section{Introduction}

Chronic exposure to ultraviolet (UV) irradiation is the leading cause of skin cancer including melanoma, squamous and basal cell carcinoma ${ }^{1,2}$. By the impingement of UV radiation, skin develops DNA photoproducts (PPs) such as cyclobutane pyrimidine dimers (CPDs) and 6-4PPs ${ }^{3}$. If such products are not eliminated by the repair mechanisms of the skin, mutations and subsequently cancers may arise ${ }^{2}$. The mechanisms that protect skin from the potential consequences of UV-induced DNA lesions involve active DNA repair by nucleotide excision, base excision and mismatch repair or as last resort induction of apoptosis and removal of cells with damages

\footnotetext{
Correspondence: Peter Wolf (peter.wolf@medunigraz.at)

${ }^{1}$ Research Unit for Photodermatology, Department of Dermatology and

Venereology, Medical University of Graz, Graz, Austria

${ }^{2}$ Center for Medical Research, Medical University of Graz, Graz, Austria

Full list of author information is available at the end of the article.

These authors contributed equally: Tej Pratap Singh, Pablo A. Vieyra-Garcia

Edited by M. Malewicz
}

in their genome $e^{3}$. Additionally, genes involved in modulation of innate and adaptive immune responses including Cbl-b, may also play an important role in the immunomodulatory and carcinogenic effects of UVB exposure $^{3-5}$. Cbl-b is a member of the mammalian $\mathrm{Cbl}$ family of proteins, a group of E3 ubiquitin ligases that consist of $\mathrm{c}-\mathrm{Cbl}, \mathrm{Cbl}-\mathrm{b}$, and $\mathrm{Cbl}-3$. Cbl-b is a negative regulator of $\mathrm{T}$-cell receptor signaling and its deficiency leads to spontaneous autoimmunity ${ }^{6,7}$. Moreover, Cbl-b is a modulator of many biological processes such as the induction of immune tolerance and antitumor immunity ${ }^{8}$. In addition, it has been demonstrated that Cbl-b-deficient mice developed fewer UVB-induced skin malignancies by spontaneously rejecting tumor cells in a $\mathrm{CD} 8^{+} \mathrm{T}$-cell and NK-cell dependent manner ${ }^{4,6,7}$. Nevertheless, the role of Cbl-b in the early events that follow UVB exposure (relevant for the subsequent carcinogenic effect of sunlight) has not been evaluated. In the present work, we

\section{(c) The Author(s) 2018}

(c) (i) Open Access This article is licensed under a Creative Commons Attribution 4.0 International License, which permits use, sharing, adaptation, distribution and reproduction c. in any medium or format, as long as you give appropriate credit to the original author(s) and the source, provide a link to the Creative Commons license, and indicate if changes were made. The images or other third party material in this article are included in the article's Creative Commons license, unless indicated otherwise in a credit line to the material. If material is not included in the article's Creative Commons license and your intended use is not permitted by statutory regulation or exceeds the permitted use, you will need to obtain permission directly from the copyright holder. To view a copy of this license, visit http://creativecommons.org/licenses/by/4.0/. 
irradiated $\mathrm{Cbl}-\mathrm{b}^{-1-}$ and wild-type (WT) mice with UVB to study the early responses of exposed skin in the absence of this ubiquitin ligase.

\section{Results}

Cbl-b-/- mice carry fewer UVB-induced SBCs and DNA PPs

Cbl-b participates in the rejection of UVB-induced tumor cells by enhancing cytotoxic immune responses mediated by tumor specific $\mathrm{CD}^{+}$cells ${ }^{9}$. We hypothesized that upon UVB irradiation, Cbl-b is highly upregulated and participates in the immunomodulatory effects of UVB in exposed skin. To evaluate the expression of $\mathrm{Cbl}-\mathrm{b}$ in response to UVB, WT mice were irradiated with $80 \mathrm{~mJ} / \mathrm{cm}^{2}$ of UVB (Fig. 1a). Samples of dorsal skin taken $24 \mathrm{~h}$ after UV exposure showed that cells mainly from the superficial dermis expressed cytoplasmic Cbl-b (Fig. 1b). Moreover, human skin irradiated with twice the minimal erythema dose of UVB also had $\mathrm{Cbl}^{-} \mathrm{b}^{+}$cells infiltrating the superficial layer of the dermis (Suppl. Fig. 1). One of the early consequences of toxic UV exposure is the formation of SBCs. A SBC is a damaged epidermal cell undergoing apoptosis characterized by a pyknotic nucleus and condensed cytoplasm ${ }^{10,11}$. To investigate the role of Cbl-b in acute UVB-induced cytotoxicity, WT and $\mathrm{Cbl}^{-b^{-1}}$ UVB-irradiated mice were sacrificed at different time points to analyse epidermal thickness, SBCs, and CPDs. Cbl- $\mathrm{b}^{-1-}$ mice showed less epidermal thickening during the first $24 \mathrm{~h}$ after UVB exposure (Fig. 1c). We observed that $6 \mathrm{~h}$ after UVB irradiation, both WT and $\mathrm{Cbl}^{-\mathrm{b}^{-1-}}$ mice had a similar number of SBCs (Suppl. Fig. 2C). However, after $24 \mathrm{~h}$, the skin of $\mathrm{Cbl}_{-} \mathrm{b}^{-1-}$ mice developed fewer SBCs compared to WT mice (i.e., 20 vs. 33 SBCs per microscopic field) (Fig. 1d, e). To evaluate the degree of DNA damage in UVB-irradiated skin, we carried out staining of thymine dimers, a specific type of CPD. Twenty-four hour after UVB irradiation; the skin of $\mathrm{Cbl}^{-\mathrm{b}^{-1-}}$ mice had significantly fewer CPDs compared to the skin of WT mice (Fig. 1f-h). To rule out differences of skin pigmentation between $\mathrm{Cbl}^{-\mathrm{b}^{-1-}}$ and WT mice (that may influence the number of SBCs and CPD in tissue) prior UVB exposure, we quantified pigmentation by noninvasive skin reflectance spectroscopy and the levels of melanin by Fontana-Masson staining and there was no influence of Cbl-b deficiency on skin pigmentation (Suppl. Fig. 2A, B). Together, these findings indicate that Cbl-b is an element of response to UVB expressed in cells of the dermis of mice and humans. Cbl-b plays a role in physiologic clearance of SBCs and its deficiency may provide protection from UVB exposure in a nonpigment-related manner.

\section{Cbl-b deficiency induces expression of epidermal growth- related genes and Wnt signaling in response to UVB irradiation}

To gain insight of the gene expression profile that may be involved in protection against DNA damage induced by UVB irradiation in $\mathrm{Cbl}^{-\mathrm{b}^{-1-}}$ mice, we isolated RNA from skin samples taken before and $24 \mathrm{~h}$ after UVB irradiation of Cbl- $\mathrm{b}^{-1-}$ and WT mice to carry out microarray analyses. We established a cut off threshold of $p$ value under 0.05 and a fold change level of \pm 1.5 to look for significant changes in gene expression. By doing so, we found 94 genes differentially regulated in $\mathrm{Cbl}^{-b^{-1-}}$ vs. WT mice after UVB exposure (Fig. 2a). There was an overexpression of several genes involved in negative regulation of apoptosis (Wisp2, Fmod, Nceh1, Cygb, and Nnt $)^{12-16}$, tumor suppression (Nr4a1, Sox15, and Msap1) ${ }^{17,18}$, oxidative-stress response (Atp10d, Cxcl11, $\mathrm{Nnt}$, and Cygb) ${ }^{12,16,19}$, tissue remodeling $(\mathrm{Mmp} 12)^{20}$, and cell survival (Tlr6, Nr1d1, Tpsb2, IL-22r1, Nr4a1, Prox1, and Fam198a) $)^{21-23}$ in UVB-exposed Cbl-b ${ }^{-1-}$ compared to WT mice. Many tumor-related (Tiam2, Tspan6, Hmgn5, Osr1, Pdcd4, Cntfr, and Vaultrc5) ${ }^{24,25}$ and inflammation-related (Saa3 and Defb8) ${ }^{26}$ genes were downregulated. A gene interaction network showed that within the upregulated genes after UVB irradiation, a cluster of five genes (Mmp12, Nr4a1, Prox1, Flt4, and F3) was directly associated to regulation of epithelial cell proliferation (Fig. 2b).

To understand whether the differentially expressed genes found in $\mathrm{Cbl}_{-} \mathrm{b}^{-1-}$ mice resulted from a direct response to UVB irradiation or a background alteration of Cbl-b deficiency, we carried out microarray analyses of the skin from nonirradiated $\mathrm{Cbl}^{-\mathrm{b}^{-1-}}$ and WT control mice (Fig. 2c). We observed that Cbl-b deficiency resulted in nine genes with differential expression as background, we plotted together the fold change values of expression from these genes comparing $\mathrm{Cbl}^{-\mathrm{b}^{-/-}}$vs. WT mice of nonirradiated and UVB-irradiated animals (Fig. 2d). Three of these nine genes (Saa3, Defb8, and Nrd1) showed at least a twofold difference of expression after UVB irradiation between irradiated and nonirradiated animals, whereas the other six genes had minor fold change variations. Saa3 flipped from a 2.57 -fold positive ratio to a 4.45 -fold negative ratio; Nr1d1 flipped from a 2.97 -fold negative to a positive 1.66 -fold ratio; Defb8 decreased from a 2.14-fold negative ratio to a 5.38-fold negative ratio comparing nonirradiated vs. UVBirradiated mice. We did not observe alterations in the expression of genes that are directly involved in DNA repair processes such as NER or BER between $\mathrm{Cbl}^{-\mathrm{b}^{-1-}}$ vs. WT mice after UVB irradiation, however, immunohistochemical stainings revealed that $\mathrm{Cbl}^{-\mathrm{b}^{-1-}}$ mice had higher expression of IL-10 24 h after exposure suggesting an increased immunomodulatory effect of UVB in these mice (Suppl. Fig. 3). Our data suggest that in response to UVB, cellular pathways involved in cell proliferation, activation, inflammation, invasion, and migration were greatly affected by the lack of Cbl-b (Suppl. Table 1). 


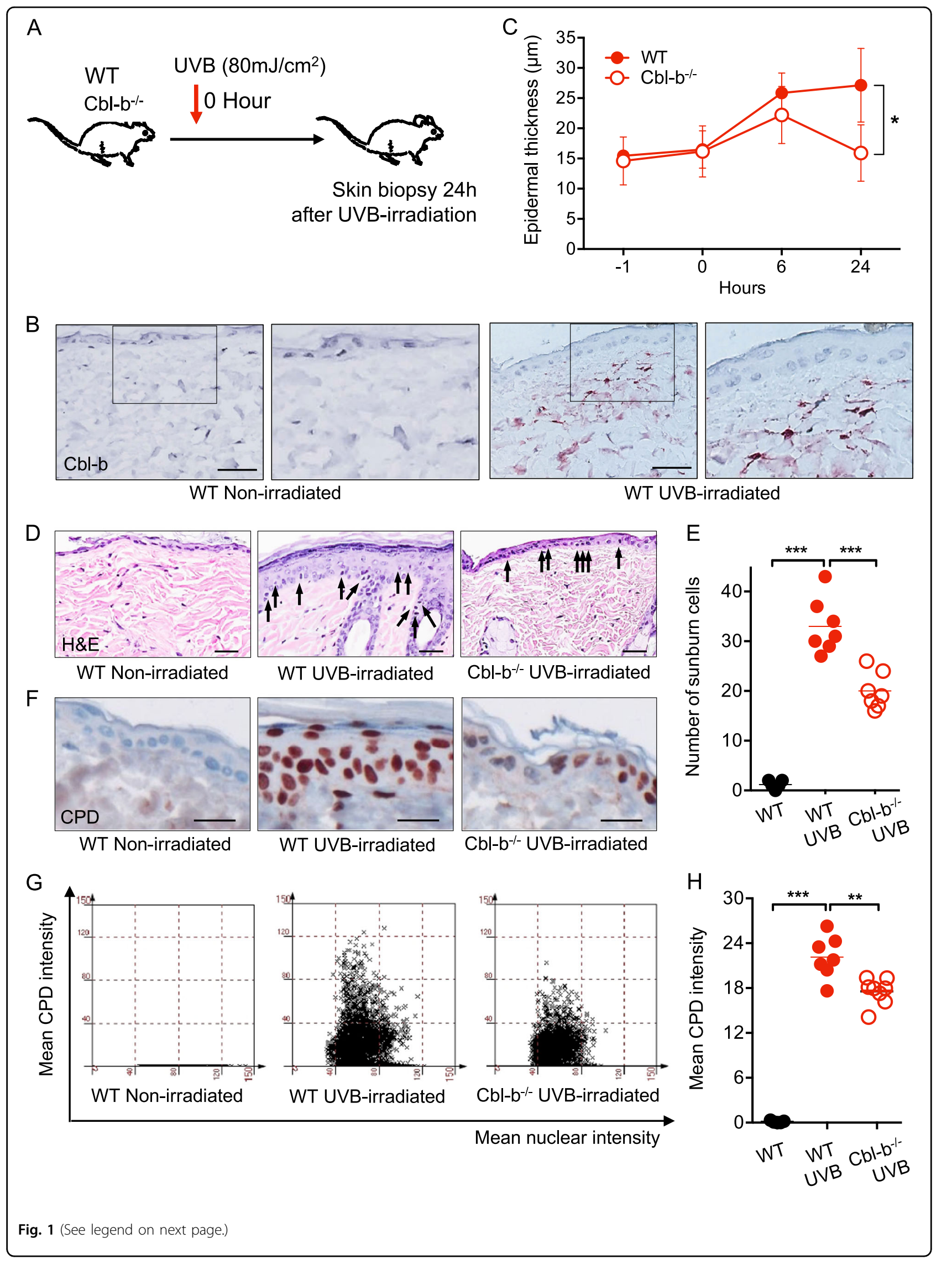


(see figure on previous page)

Fig. $1 \mathrm{Cbl-b^{-1 }}$ mice are protected from UVB-induced sunburn cell formation and DNA damage. a Schematic diagram showing the UVB irradiation protocol to induce short-term cytotoxicity. WT and $\mathrm{Cbl}_{-1} \mathrm{~b}^{-1-}$ mice were sacrificed at $24 \mathrm{~h}$ after the UVB exposure and dorsal skin samples were collected for SBC and CPD evaluation. b Cbl-b staining of dorsal skin sections of WT mice before and $24 \mathrm{~h}$ after UVB irradiation. Scale bar $50 \mu \mathrm{m}$. c Mean epidermal thickness during the first $24 \mathrm{~h}$ after UVB irradiation of WT and $\mathrm{Cbl}-\mathrm{b}^{-1-}$ mice. Five mice were used per group, the experiment was done in three independent replicates. Representative data from three independent experiments. $n=5$ mice per group. ${ }^{*} P<0.05$; error bar is SD. d Representative H\&E sections of dorsal skin to evaluate the number of SBCs. Arrows depict SBCs. e Averaged numbers of SBCs per field and their group mean in H\&E sections of dorsal skin. $n=5-7$ mice per group; ${ }^{* *} P<0.001$. f Representative micrograph of CPD antibody-stained sections of dorsal skin to evaluate CPDs formation. $\mathbf{g}$ CPD antibody-stained sections of dorsal skin were analysed by tissue cell analysis. Representative plot of intensity of CPD- stained cells ( $Y$-axis) and nuclear intensity ( $X$-axis) for quantification of CPDs formation in epidermis of one mouse from each group. h Mean relative intensity of CPD positivity in epidermis of dorsal skin of individual mice. $n=5-7$ mice per group; ${ }^{* *} P<0.01,{ }^{* * *} P<0.001$

Among upregulated genes we found several members of the Wnt signaling pathway like Sox15, Wisp2, Nr4a1, Prox1, and Mmp12 $2^{27-29}$. Therefore, we stained UVBirradiated skin for $\beta$-catenin and MMP12 to evaluate Wnt activation and found a high number of $\beta$-catenin ${ }^{+}$and $\mathrm{MMP}^{+}$cells in dermis and epidermis of $\mathrm{Cbl}^{-\mathrm{b}^{-/-}}$ compared to WT mice (Fig. 3a, b), suggesting an activation of Wnt signaling and a possible participation in tissue remodeling and removal of SBCs. Altogether, these results indicate that $\mathrm{Cbl}_{-} \mathrm{b}^{-1-}$ mice have differential transcriptional profile in UVB-irradiated skin that includes some genes from the background of Cbl-b deficiency, playing a role in regulation of cell proliferation and tumor/inflammation inhibition. Moreover, $\beta$-catenin activation suggests that Wnt signaling may crosstalk with Cbl-b as previous findings have suggested ${ }^{30}$.

\section{$\mathrm{Cbl}^{-\mathrm{b}^{-1-}}$ mice show lower degree of UVB-induced inflammation and epidermal hyperplasia}

Chronic inflammation is known to be a promotion factor in photocarcinogenesis. We tested whether deficiency of Cbl-b protects the skin from chronic inflammation. The dorsal skin of $\mathrm{WT}$ and $\mathrm{Cbl}-\mathrm{b}^{-1-}$ mice was exposed to 6 doses of $80 \mathrm{~mJ} / \mathrm{cm}^{2}$ and subsequent 6 doses of $220 \mathrm{~mJ} / \mathrm{cm}^{2}$ during 23 days as described in Fig. $4 \mathrm{a}$. Macroscopic double skin fold thickness (DSFT) and microscopic epidermal thickness were measured over time as readout of inflammatory response. $\mathrm{Cbl}^{-\mathrm{b}^{-/-}}$, but not WT mice were protected from chronic inflammation evidenced by a lower DSFT from the first week throughout the entire period of the UVB irradiation protocol (Fig. 4b). Epidermal thickening was reduced in UVB-irradiated Cbl- $\mathrm{b}^{-1-}$ mice compared to WT by the end of the UVB exposure protocol (Fig. 4c).

UVB-induced immunosuppression is crucial in the promotion of skin cancer. $\mathrm{CD} 4{ }^{+} \mathrm{CD} 25^{+} \mathrm{Foxp} 3^{+}$regulatory $\mathrm{T}$ cells (Tregs) secreting IL-10 play an important role in the effect of UVB and participate in immune tolerance to UV-generated malignant cells ${ }^{10}$. After repetitive exposure to UVB we observed no significant changes in Tregs in the skin-draining lymph nodes of $\mathrm{Cbl}^{-\mathrm{b}^{-/-}}$compared to WT mice (not shown). However, the expression of IL-10 was

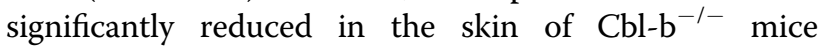
(Fig. 4d). These results suggest that Cbl-b participates in sustaining chronic inflammation without interfering in the induction and/or recruitment of Tregs. Nonetheless, regulatory cytokines like IL-10 could be produced under Cbl-b regulation, thus, a lack of Cbl-b expression may abolish some of the immunomodulatory effects of UVB.

\section{Discussion}

The regulatory function of Cbl-b has been explored in lymphocyte activation, autoimmunity and carcinogenesis. UVB-induced tumors are rejected in $\mathrm{Cbl}^{-\mathrm{b}^{-1-}}$ mice by an efficient activation of tumor specific cytotoxic $\mathrm{CD}^{+}$ $\mathrm{T}$ cells ${ }^{6}$. In this study we addressed the immediate response to UVB of $\mathrm{Cbl}^{-\mathrm{b}^{-1-}}$ mice to understand the early events that may lead to the improved tumor immunity previously observed in these mice ${ }^{6}$. Irradiation of human skin showed infiltration of Cbl-b ${ }^{+}$cells (Suppl. Fig. 1)suggesting that this ubiquitin ligase may have a relevant role in the effects of UVB such as carcinogenesis associated to excessive sun exposure. $\mathrm{Cbl}^{-b^{+}}$cells infiltrated the skin of WT mice after UVB exposure (Fig. 1b), possibly indirectly affecting removal of SBCs and CPDs in epidermis. In fact, the response to UVB-induced DNA damage can be modulated by inflammatory cytokines such as IL-10 and IL-18 ${ }^{31,32}$. IL-10 staining $24 \mathrm{~h}$ after UVB exposure (Suppl. Fig. 3) demonstrated that Cbl-b deficiency resulted in a higher expression of this cytokine but after chronic exposure for up to 4 weeks and 12 sessions, qPCR results (Fig. 4d) showed a drastic drop in the expression of IL-10 in Cbl-b ${ }^{-1-}$ mice. Controversially, microarray results (Fig. 2a) and staining of XPC (not shown) did not indicate alterations in DNA repair machinery in $\mathrm{Cbl}^{-\mathrm{b}^{-1-}}$ mice although previous investigations have associated UVB-induced DNA damage with high levels of $\mathrm{IL}-10^{33}$. Interestingly, Cbl-b deficient effector $\mathrm{T}$ cells are resistant from Treg/IL-10 mediated suppression. Nevertheless, despite the immunosuppressive environment of the skin, Cbl-b deficiency promoted the clearance of SBCs. 

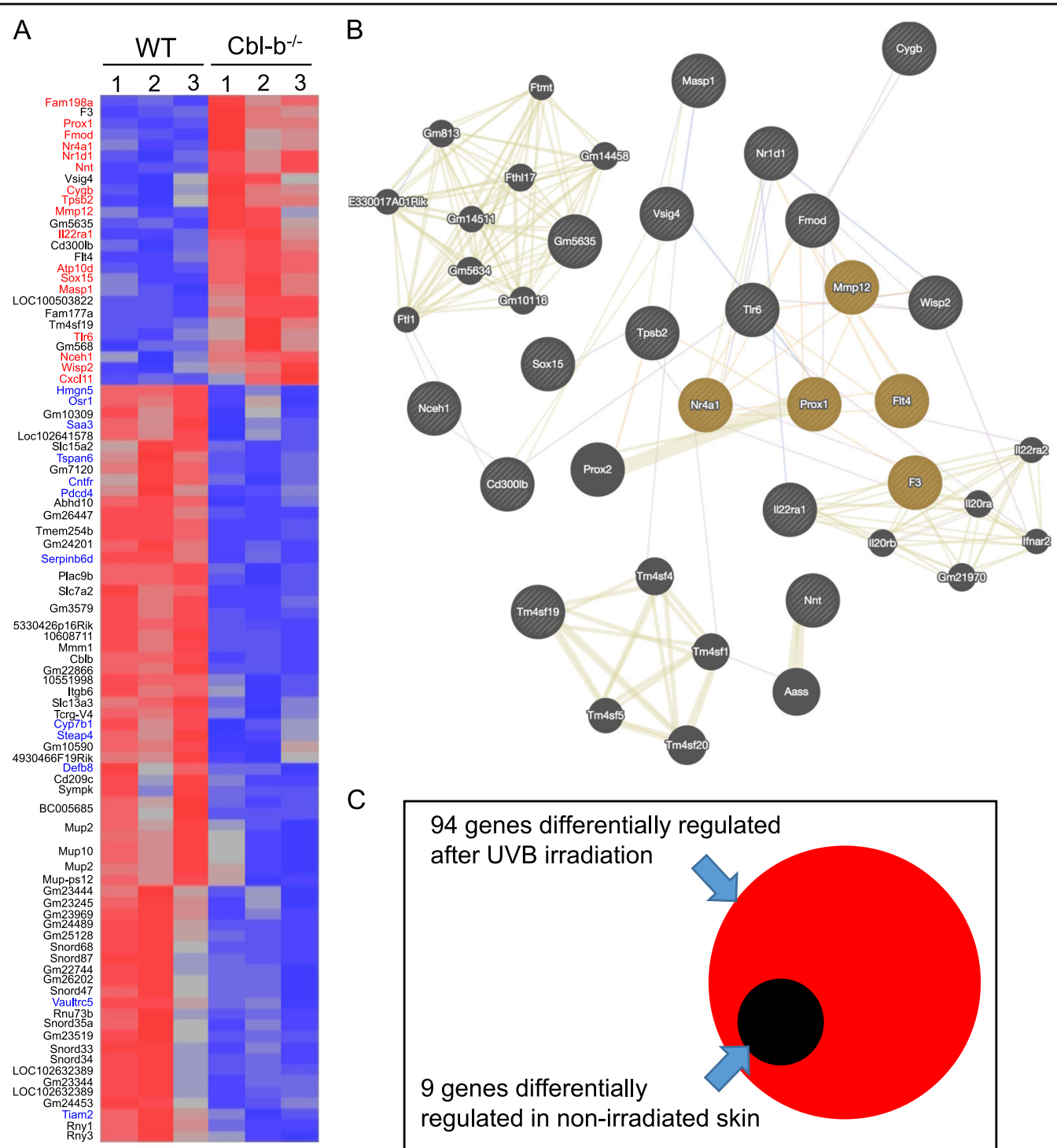

C
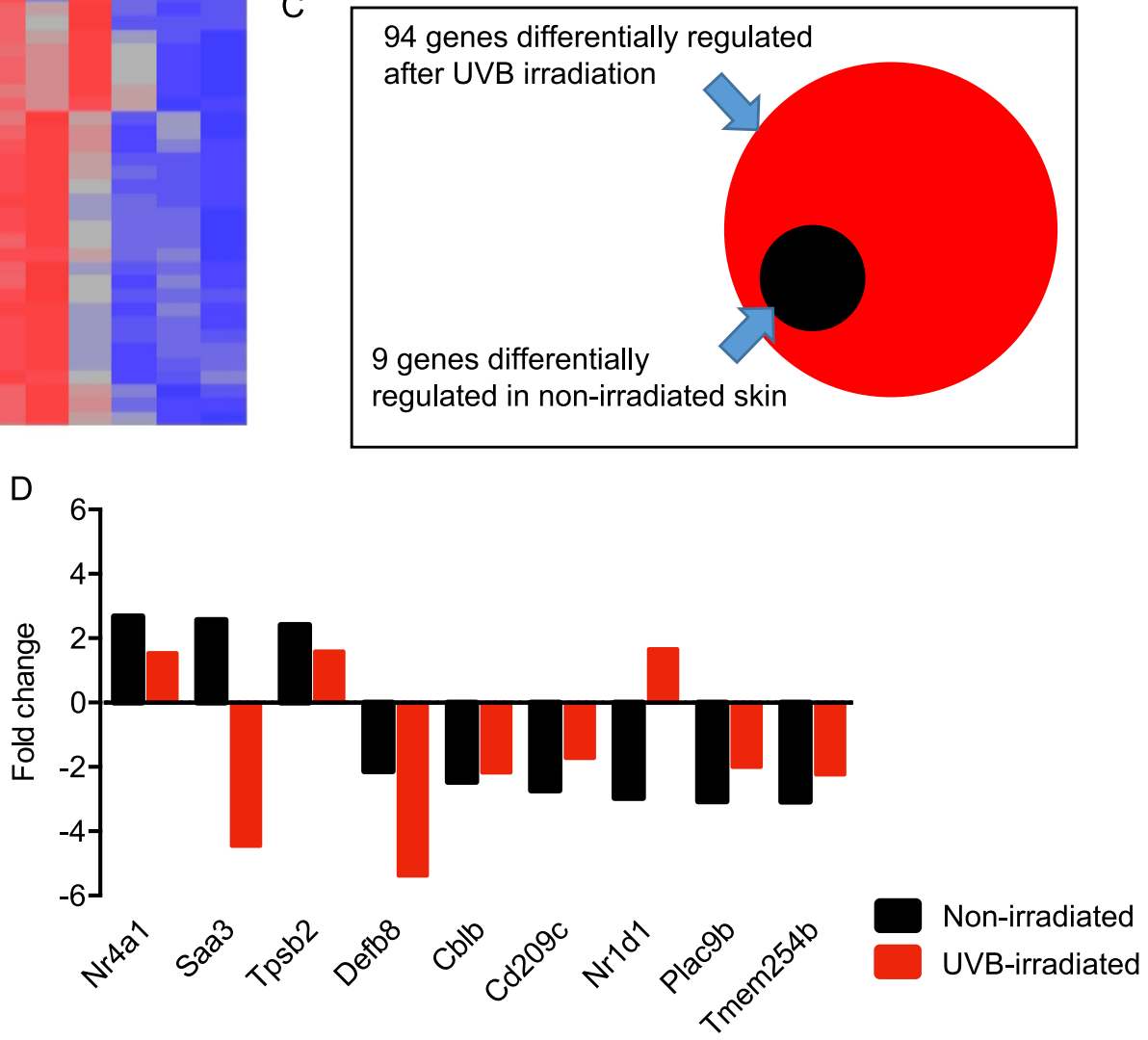

Fig. 2 (See legend on next page.) 
(see figure on previous page)

Fig. 2 Gene expression analyzes on UVB-irradiated mice. WT and $\mathrm{Cbl}_{-1} \mathrm{~b}^{-1-}$ mice were sacrificed at $24 \mathrm{~h}$ after a single UVB exposure. Irradiated skin was excised to isolate RNA for microarray analysis (Affymetrix Mouse Gene 1.0 ST Array) and for immunostaining. a Heat map showing differentially expressed genes in $\mathrm{Cbl}_{-} \mathrm{b}^{-/-}$compared to WT mice ( $n=3$ mice per group). Ninety-four genes were found deregulated as defined by a $p$ value $<0.05$ and \pm 1.5 -fold change as cut off. Highest to lowest gene expression is depicted as red to blue color code in the heat map (Partek Genomics Suite 6.5). Gene names marked in red and blue are discussed in the Results section. $\mathbf{b}$ Gene interaction network of upregulated genes (large circles). A cluster of genes associated to positive regulation of epithelial cell proliferation was found ( $F D R=0.0042$, brown circles). A prediction of upregulated genes by shared domain (medium circles) or coexpression (small circles) was done with GeneMANIA webserver. Nodes are connected by co-localization (blue lines) and predicted/shared protein domains (brown lines). c Overlapping of differentially expressed genes in skin of $\mathrm{Cbl}_{-} \mathrm{b}^{-/-}$vs. WT mice before (black) and after UVB irradiation (red). d Fold change comparison of nine genes with preexisting differential expression in nonirradiated and UVBirradiated mice. Fold changes in $\mathrm{Cbl}_{-} \mathrm{b}^{-/-}$vs. WT are shown in the waterfall plot

After exposure to UVB, Cbl-b ${ }^{-1-}$ mice showed a reduced epidermal thickening compared to WT mice, suggesting a lower degree of overall cell injury (Fig. 1c). The quantification of CPDs and SBCs provides an accurate assessment of the damage in irradiated tissue and may predict the risk of tumor development. Indeed, SBCs are considered a surrogate marker of UV-induced DNA damage to the $\operatorname{skin}^{34}$. By using two different protocols of UVB exposure we studied the effects of Cbl-b deficiency on SBCs and CPDs formation and inflammation. We found that $24 \mathrm{~h}$ after exposure to a single dose of UVB, the skin of Cbl-b ${ }^{-1-}$ mice developed fewer SBCs and DNA dimers (Fig. 1c-h). The peak of UVB acute effects are regularly observed between 6 and $24 \mathrm{~h}$, depending on UVB wavelength and dose $\mathrm{s}^{35,36}$. SBC are visible as early as 30 min after irradiation and reach their highest in numbers within $24 \mathrm{~h}^{37}$, offering with the first $24 \mathrm{~h}$ after exposure a well delimited time-span to study immediate effects of UVB. The relevance of these early events post UVB irradiation are highlighted by studies in which the application of topical DNA repair enzymes reverts suppression in induction of contact hypersensitivity and protects from Langerhans cell depletion in UV-irradiated mice $^{38}$. Moreover, DNA repair-deficient $\mathrm{XP}^{39}$ and IL$12 \mathrm{p} 40^{-1-}$ mice exhibited an increased number of SBCs compared to WT after UVB irradiation ${ }^{34,40}$. Our results indicate that DNA PPs and SBCs are linked to Cbl-b expression, suggesting that Cbl-b may be involved directly or indirectly in controlling DNA repair mechanisms. Alternatively, the lack of Cbl-b may result in an enhanced removal of cells carrying DNA damage by an efficient cytotoxic activity of $\mathrm{CD}^{+}$and NK cells as demonstrated by previous results ${ }^{6,7}$. Moreover, we found no indication of a gains or losses in the mechanisms that cope with detoxification of ROS and DNA damage in $\mathrm{Cbl}^{-\mathrm{b}^{-1-}}$ mice since staining of 8-hydroxyguanosine, XPC and 6,4-PPs did not differ between WT vs. Cbl- $\mathrm{b}^{-1-}$ mice (not shown). Having said so, it is known that Cbl-b carries a UBA domain shared with the DNA repair protein $\operatorname{Rad} 23$, however, the functional role of the Cbl-b UBA domain is not known ${ }^{41}$.

The transcriptional profile of WT compared to $\mathrm{Cbl}_{-} \mathrm{b}^{-1-}$ mice after UVB irradiation showed 85 differentially regulated genes in addition to 9 genes with background differential expression in nonirradiated mice. Ubiquitin ligases like $\mathrm{Cbl}-\mathrm{b}$ are known negative regulators of tyrosine kinase signaling. Their regulatory effect in cells of the immune system has been largely described ${ }^{42}$ and more recently, Cbl-b has been implicated in the maintenance of mammary stem cell phenotype involving a negative regulation of the AKT-mTOR pathway ${ }^{43}$. Our results suggest that Cbl-b deficiency may affect the transcriptional profile by shifting the activation of Wnt and tyrosine kinase signaling. The genes affected by UVB irradiation play a role in regulation of cellular processes like cell cycle, cell adhesion, invasion, and migration. It is known that Wnt signaling orchestrates DNA damage response through rescue of irradiated cells from apoptosis in a process regulated by c-Cbl targeting $\beta$-catenin ${ }^{44,45}$, a similar mechanism could also operate with Cbl-b. This observation can be supported by the presence of high number of $\beta$-catenin positive cells in UVB-irradiated Cbl$\mathrm{b}^{-1-}$ mice (Fig. 3a). Although, $\beta$-catenin was upregulated after UVB irradiation together with other epidermal cell proliferation regulators, skin thickness of $\mathrm{Cbl}^{-b^{-/-}}$mice was lower compared to control WT mice after chronic exposure to UVB (Fig. $3 \mathrm{~b}$ ), suggesting that Cbl-b may limit the mechanisms that ameliorate UV damage to exposed cells and participate in the early events of phototoxicity and subsequent carcinogenesis. Moreover, metalloproteinases are enzymes that mediate photoaging and tissue remodeling ${ }^{46}$. Some metalloproteinases like MMP13 are negatively regulated by $\mathrm{Cbl}^{-b^{47}}$. UVBirradiated $\mathrm{Cbl}^{-\mathrm{b}^{-1-}}$ mice had an increase of MMP12 expression in cells of the epidermis (Fig. 3b), suggesting that upon UVB these mice may have a higher rate of tissue remodeling and elimination of SBC.

Excessive exposure to UVB is a known promotion factor of carcinogenesis. Our data indicate that Cbl-b deficiency 

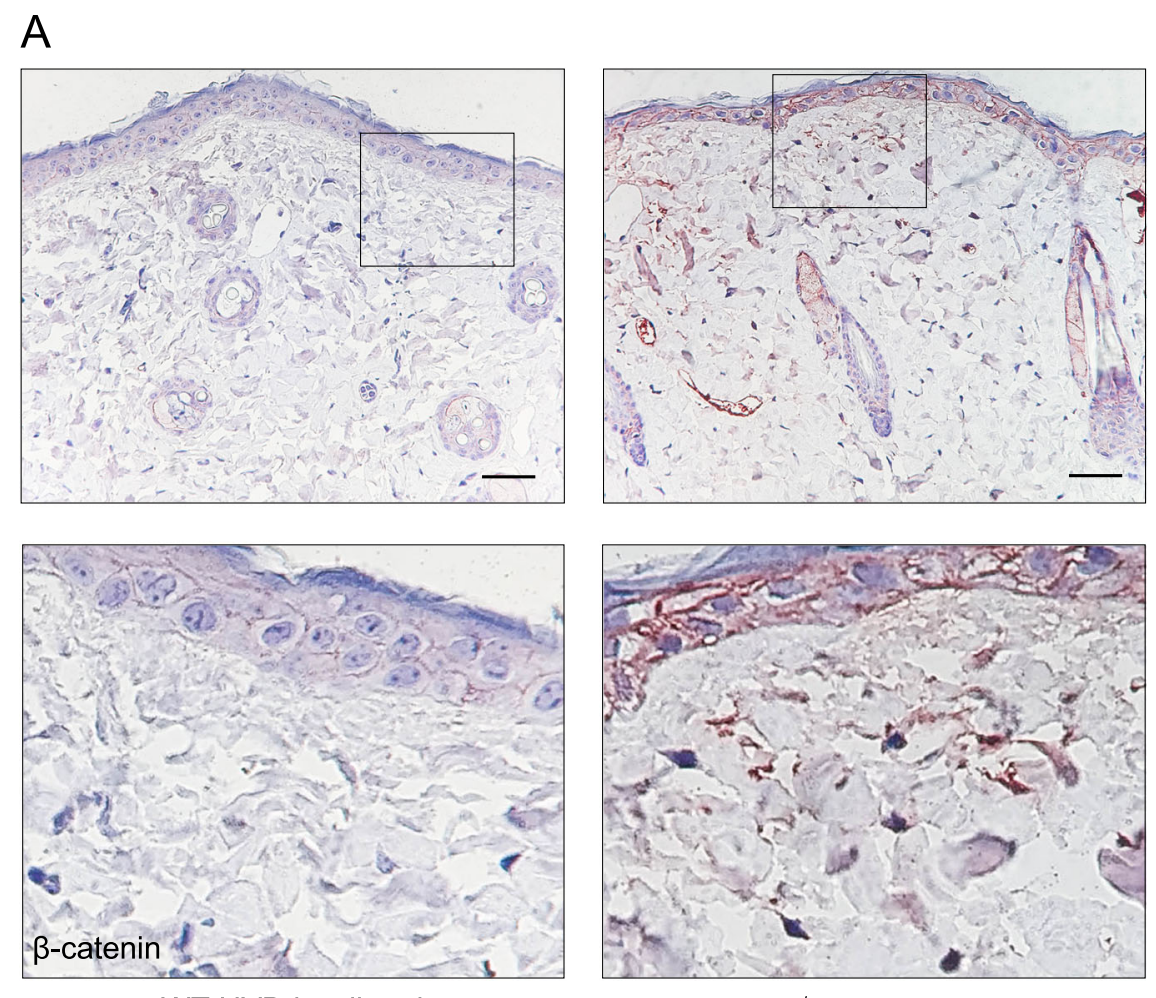

WT UVB-irradiated

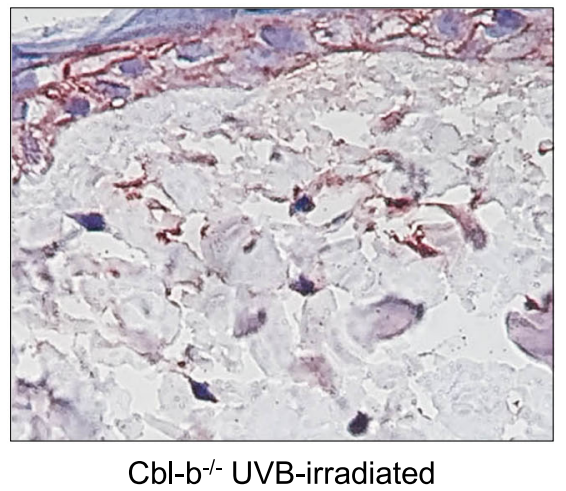

B
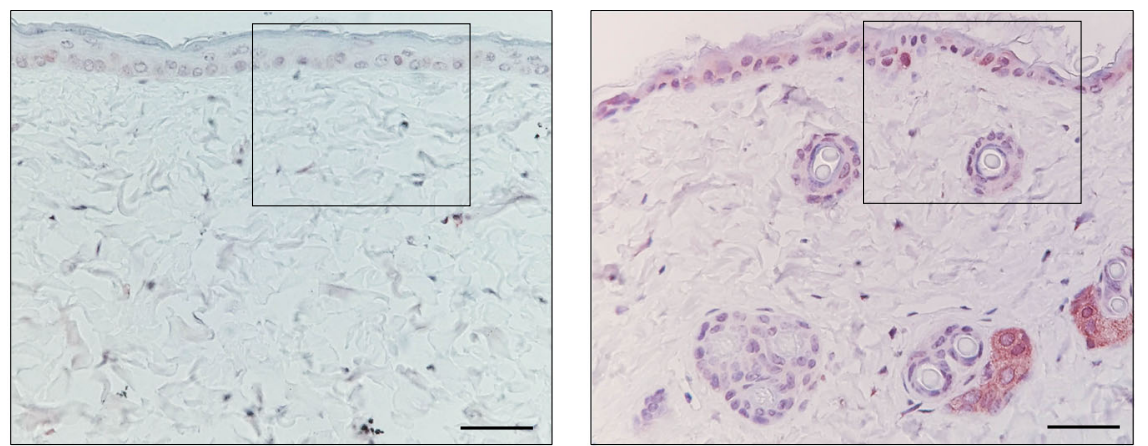

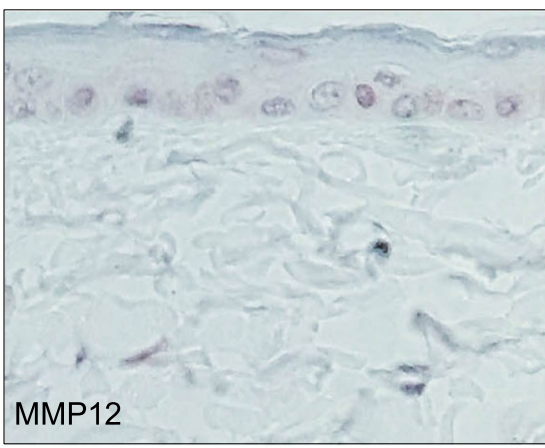

WT UVB-irradiated

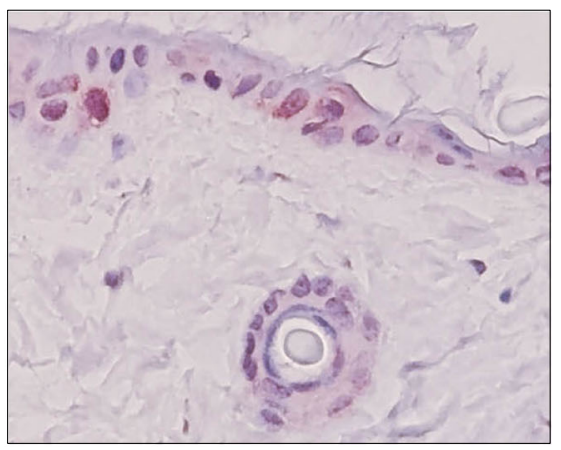

Cbl-b-/- UVB-irradiated

Fig. $3 \mathrm{Cbl-b^{-1- }}$ mice express $\beta$-catenin and MMP12 after UVB irradiation. Representative photographs from paraffin sections of dorsal skin of WT or $\mathrm{Cbl}^{-\mathrm{b}^{-1}-}$ mice at $24 \mathrm{~h}$ after UVB irradiation stained with $\beta$-catenin (a) and MMP12 (b). Marked squares of the upper panel are shown at higher magnification in the lower panel. Scale bar $50 \mu \mathrm{m}$ 

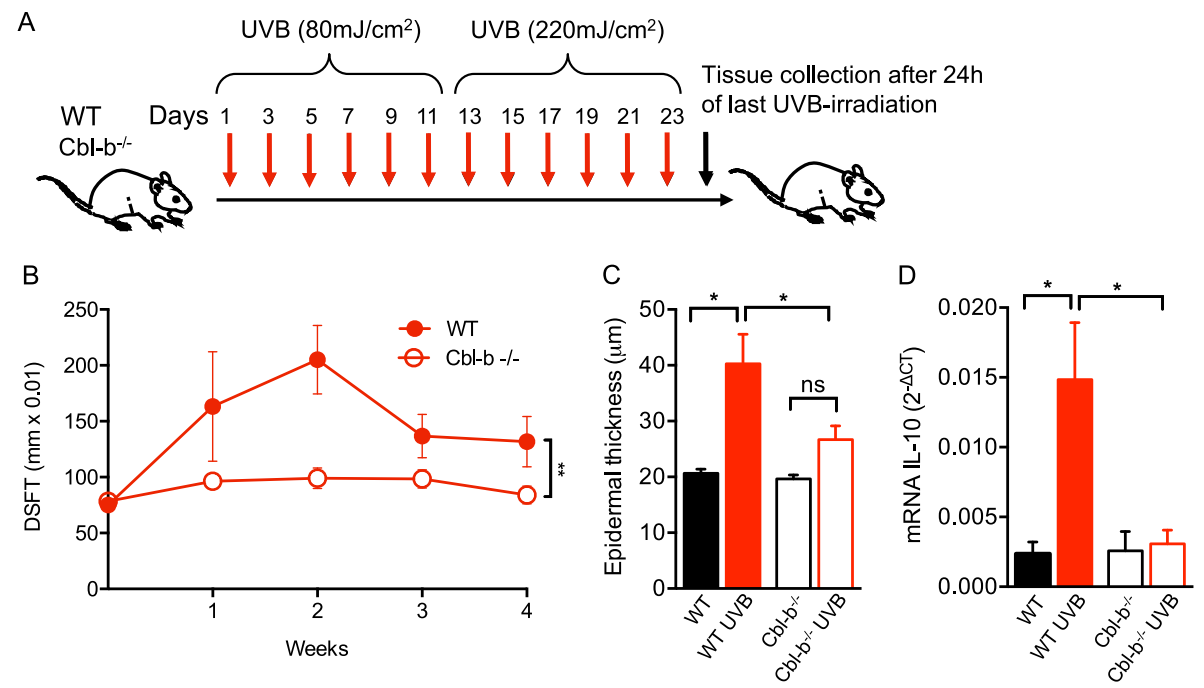

Fig. $4 \mathrm{Cbl-b^{-/ }}$ mice are protected from UVB-induced chronic inflammation. a Schematic diagram showing the UVB irradiation protocol to induce chronic inflammation. WT and Cbl- $\mathrm{b}^{-1-}$ mice were sacrificed at $24 \mathrm{~h}$ after the last UVB exposure. Dorsal skin was collected for analysis. $\mathbf{b}$ Mean double skin fold thickness (DSFT) of dorsal skin of WT and $\mathrm{Cbl}_{-1} \mathrm{~b}^{-1-}$ mice throughout 4 weeks of the UVB irradiation protocol. $n=5-7$ mice per group. ${ }^{* *} P<$ 0.01; error bar is SD. c Epidermal thickness of H\&E-stained sections $24 \mathrm{~h}$ after last UVB exposure (at day 24$) . n=5-7$ mice per group. ${ }^{*} P<0.05$; error bar is SEM. $\mathbf{d}$ qRT-PCR analysis of IL-10 in dorsal skin. $n=5-7$ mice per group. ${ }^{*} P<0.05$; error bar is SEM

protects from some of the effects of repetitive exposure to UVB as measured by lower cellular skin infiltration, swelling, and epidermal hyperplasia (Fig. 4b, c). Despite the low dose of UVB and the short period of irradiation used in our experiments (in comparison with studies of UVB tumor models ${ }^{48}$ ), IL-10 (a known mediator of UVBinduced immunosuppression ${ }^{2,5,8,10}$ ) was initially upregulated $24 \mathrm{~h}$ after a single exposure to UVB. Chronic exposure to UVB led to an increased expression of IL-10 in skin of WT mice, however, $\mathrm{Cbl}^{-\mathrm{b}^{-l-}}$ mice failed to upregulate this cytokine and showed lower levels of IL-10 (Fig. 4d). Animal models of photocarcinogenesis have demonstrated that regulatory $\mathrm{T}$ cells mediate a large proportion of the immunosuppressive effects of UVB with a peaks of infiltration in irradiated skin 7 days after initial exposure and subsequent normalization ${ }^{49}$. Nonetheless, our study was consistent with previous findings of unchanged Treg numbers in the skin after exposure of $\mathrm{Cbl}_{-} \mathrm{b}^{-1-}$ mice to UVB and a possible higher cytotoxic activity of $\mathrm{CD}^{+} \mathrm{T}$ cells by a decreased sensitivity to Tregmediated immunosuppression ${ }^{6}$. Whether the origin of the observed protective $\mathrm{Cbl- \textrm {b } ^ { - 1 - }}$ phenotype is due to the decreased DNA damage in the epidermis, an autocrine effect or via interaction with the altered immune system in these mice remains to be determined.

In summary, UVB irradiation in Cbl-b deficient mice leads to a lower number of DNA PPs and SBCs, higher expression of Wnt signaling mediators like $\beta$-catenin and a differential gene expression signature enriched with cell proliferation regulators compared to WT control mice. This suggests that Cbl-b is involved in the immediate response to UVB cytotoxicity. Thus, if cells can indeed better tolerate UVB and survive in the absence of Cbl-b (without harboring internal damage), then our findings may open new avenues to target this gene in novel sun protection strategies.

\section{Materials and methods \\ Animals}

BL6 WT and $\mathrm{Cbl}^{-\mathrm{b}^{-/-}}$mice were obtained from the Institute of Molecular Biotechnology (IMBA) Vienna, Austria and held in our facility at the Medical University of Graz. All animals were maintained with alternating $12 \mathrm{~h}$ light and dark cycles, as well as controlled temperature and humidity in our facilities. Mice were shaved on the dorsal skin 1 day prior experimental procedures. All animal procedures were approved by the Federal Ministry of Science and Research, Austrian Government through protocol no. BMWF-66.010/0019-II/3b/2011. All methods were performed in accordance with the relevant guidelines and regulations.

\section{UVB irradiation of animals}

Mice were exposed to a single dose of $80 \mathrm{~mJ} / \mathrm{cm}^{2}$ of UVB. A Waldmann UV236B irradiation system equipped with two fluorescent CF-L 36 W/UV6 light tubes (emission range, $280-360 \mathrm{~nm}$; peak, $324 \mathrm{~nm}$; Waldmann Medizintechnik, Villingen-Schwenningen, Germany) was used for UVB irradiation, at a mean irradiance of $2.20 \mathrm{~mW} / \mathrm{cm}^{2}$ at a distance of $15 \mathrm{~cm}$ and the irradiance of exposure was monitored by a calibrated Waldmann photometer. The applied UVB dose corresponded to 
approximately one minimal erythema dose, as determined by exposure to a series of UVB doses at the increments by factor of 1.4 , as previously described ${ }^{50}$. To study the chronic effects, mice were exposed to repetitive UVB doses on alternate days for 23 days at a dose of $80 \mathrm{~mJ} / \mathrm{cm}^{2}$ for 11 days and then at a dose of $220 \mathrm{~mJ} / \mathrm{cm}^{2}$ from day 12 to 23 by using the same irradiation system. Skin inflammation was monitored by measuring the DSFT of dorsal skin with an engineer's micrometer (Mitutoyo Corporation) and mice were killed 6 or $24 \mathrm{~h}$ after last UVB exposure for tissue collection. Noninvasive skin pigmentation was determined by skin reflectance spectroscopy on shaved dorsal skin with a DermaSpectometer (Cortex Technologies, DK).

\section{Humans skin samples}

Paraffin-embedded materials were available for immunohistochemical stainings from a previous clinical study. In accordance with the study protocol (IRB approval number: 15-129 ex 93/94) healthy volunteers had been irradiated on their buttocks with solar simulated UV radiation equivalent to two times of the minimal erythema dose and skin biopsies had been taken $24 \mathrm{~h}$ after exposure.

\section{Histological evaluation}

Epidermal hyperplasia was assessed on H\&E-stained sections of dorsal skin by measuring the histologic thickness of the epidermis. SBCs were counted in the interfollicular epidermis of H\&E-stained dorsal skin sections in at least 10 random fields (at a final magnification $20 \times$ ). All measurements were performed in a blinded manner. Photographic images were acquired by using a DP71 digital camera (Olympus) attached to an Olympus BX51 microscope.

\section{Immunohistochemical and image analysis}

Skin sections of mice were pretreated with EDTA at $\mathrm{pH}$ 8 and then incubated with antithymine dimer (clone KTM53; Kamiya Biomedical Co, Seattle, WA) (1:2000), anti-MMP12 (clone EP1261Y; Abcam ab52897) anti-IL10 (clone JES5-2A5; Abcam ab189392) or anti- $\beta$-catenin monoclonal antibody (clone E247; Abcam, Oxford UK). Sections of human and mice skin were stained with anti Cbl-b (clone 246C5a; Abcam ab54362). The Dako K 5003 detection system (Dako, Glostrup, Denmark) was used for visualization according to the manufacturer's instructions. Melanin determination was performed by standard Fontana-Masson staining (Ab1506669, Abcam, Oxford, UK) according to manufacturer instructions. To quantify CPDs, antibody-stained tissue sections were scanned on TissueFAXS system by TissueFAXS cell tissue analysis software (TissueGnostics GmbH, Vienna, Austria) and the epidermis was electronically dissected with ImageJ software for intensity measurement by TissueQuest software (TissueGnostics GmbH, Vienna, Austria).

\section{Quantitative RT-PCR}

RNA was extracted from dorsal skin tissue using QIAGEN fibrous mini kit (QIAGEN) and cDNA was made by using First strand cDNA synthesis kit (Roche). Quantitative RT-PCR for IL-10 was performed on an Applied Biosystems 7900HT system by using RT. SYBR Green/ ROX qPCR Master Mix (SABiosciences). The 2- $\Delta \mathrm{Ct}$ method was used to normalize the transcript to GAPDH.

\section{Microarray analysis}

The Affymetrix Gene Chip Microarray platform was used for identifying genes that are transcriptionally influenced. We processed three biological replicates of dorsal skin RNA samples collected from WT and Cbl- $\mathrm{b}^{-1}$ - mice before and $24 \mathrm{~h}$ after exposure to a single dose of $80 \mathrm{~mJ} / \mathrm{cm}^{2}$ UVB on the Affymetrix Mouse Gene $1.0 \mathrm{ST}$ Array (Affymetrix, Santa Clara, CA, USA) to determine the transcriptional profile. Total RNA was extracted as described above and checked for quality on the BioAnalyzer BA2100 (Agilent, Foster City, CA). For amplification, $400 \mathrm{ng}$ of the total RNA was used with the Ambion Whole Transcript Expression Kit for Affymetrix GeneChip, Whole Transcript Expression Arrays (Life Technologies; Carlsbad, California). The first strand cDNA was created according to the manufacturer protocol, which then synthesized the second strand cDNA. During the following in vitro transcription, complementary RNA (cRNA) was generated. After purification, a second cycle of first strand cDNA synthesis was performed implementing dUTPs for fragmentation. RNaseH hydrolyzed the cRNA, followed by an enzymatic fragmentation and biotin-labeling (Affymetrix GeneChip Whole Transcript Terminal Labeling and Hybridization for use with Ambion Whole Transcript; Affymetrix, Santa Clara, CA, USA). We hybridized the fragmented samples overnight rotating the arrays at $60 \mathrm{rpm}$. After washing at the Affymetrix Genechip fluidics station 450, protocol FS450_0007 (Affymetrix GeneChip HT hybridization, Wash, and Stain Kit; Affymetrix, Santa Clara, CA, USA) the arrays were scanned with the Affymetrix Scanner GCS3000, AGCC (Command Console software AGCC 3.1.1) with default analysis settings for generation of CELfiles. The arrays were evaluated based on the internal array controls using Affymetrix Genexpression Console (1.1.2). Gene interaction analysis was done using the GeneMANIA webserver ${ }^{51}$. CEL-files were imported into Partek Genomic Suite v6.6 software (Partek Inc., St Louis, $\mathrm{MO})$ for performing robust multichip average normalization including background correction, quantile normalization across all arrays, and median polished summarization based on $\log$ transformed expression 
values. A fold change of \pm 1.5 in gene expression was considered as differentially relevant. To gain insight into functional processes we used Ingenuity Pathway Analysis (IPA; Qiagen, Redwood City, CA). Differentially expressed genes in $\mathrm{Cbl}_{-} \mathrm{b}^{-1-}$ vs. WT mice before and after UVB irradiation were compared and plotted in a waterfall graph. Raw data is available on the Gene Expression Omnibus (GEO) database with the dataset number GSE79073.

\section{Statistical analysis}

Data were expressed as mean \pm SEM. Statistical differences among experimental groups were determined by using two-tailed unpaired $t$ test or one-way ANOVA (for microarray analysis). Statistical significance was set at $P<0.05$.

\section{Acknowledgments}

This work was supported by the Austrian Science Fund FWF (W1241) and the Medical University of Graz through the PhD programs MolMed and Molecular Fundamentals of Inflammation (DK-MOLIN). The authors thank Melita Ticevic and Renu Sarao, Institute of Molecular biotechnology (IMBA), Vienna, Austria, and Gerlinde Mayer, Medical University of Graz, Austria for technical support. Special thanks go to Honnavara N. Ananthaswamy, The University of Texas MD Anderson Cancer Center, Houston, Texas, for critical reading and editing of the manuscript.

\section{Author details}

${ }^{1}$ Research Unit for Photodermatology, Department of Dermatology and Venereology, Medical University of Graz, Graz, Austria. ${ }^{2}$ Center for Medical Research, Medical University of Graz, Graz, Austria. ${ }^{3}$ Institute of Molecular Biotechnology (IMBA), Vienna, Austria. ${ }^{4}$ Present address: Inflammation Biology Section, Laboratory of Molecular Immunology, NIAID, NIH, Building 10, Room 11N112, 10 Center Drive, Bethesda, MD 20892, USA

\section{Author contributions}

T.P.S., P.V., and P.W. designed the study and experiments, analysed and interpreted the data and wrote the manuscript; T.P.S, P.V., and K.W. performed experiments; K.W., P.V., and T.P.S analysed data; J.P. contributed to designing experiments and editing the manuscript.

\section{Conflict of interest}

J.P. holds shares in a company that tries to develop Cbl-b blockers. The other authors declare no competing financial interests.

\section{Publisher's note}

Springer Nature remains neutral with regard to jurisdictional claims in published maps and institutional affiliations.

Supplementary Information accompanies this paper at (https://doi.org/ 10.1038/s41419-018-0858-5).

Received: 24 July 2017 Revised: 28 June 2018 Accepted: 4 July 2018 Published online: 06 August 2018

\section{References}

1. Ramos, J., Villa, J., Ruiz, A., Armstrong, R. \& Matta, J. UV dose determines key characteristics of nonmelanoma skin cancer. Cancer Epidemiol. Biomark. Prev. 13, 2006-2011 (2004).

2. Lo, J. A. \& Fisher, D. E. The melanoma revolution: from UV carcinogenesis to a new era in therapeutics. Science 346, 945-949 (2014).
3. Matsumura, Y. \& Ananthaswamy, H. N. Toxic effects of ultraviolet radiation on the skin. Toxicol. Appl. Pharmacol. 195, 298-308 (2004).

4. Chiang, J. Y., Jang, I. K., Hodes, R. \& Gu, H. Ablation of Cbl-b provides protection against transplanted and spontaneous tumors. J. Clin. Invest. 117, 1029-1036 (2007).

5. Loser, $\mathrm{K}$. et al. Epidermal RANKL controls regulatory T-cell numbers via activation of dendritic cells. Nat. Med. 12, 1372-1379 (2006).

6. Loeser, S. et al. Spontaneous tumor rejection by cbl-b-deficient CD8+ T cells. J. Exp. Med. 204, 879-891 (2007).

7. Paolino, M. et al. The E3 ligase Cbl-b and TAM receptors regulate cancer metastasis via natural killer cells. Nature 507, 508-512 (2014).

8. Lutz-Nicoladoni, C., Wolf, D. \& Sopper, S. Modulation of immune cell functions by the E3 ligase Cbl-b. Front. Oncol. 5, 58 (2015).

9. Loeser, S. \& Penninger, J. M. The ubiquitin E3 ligase Cbl-b in T cells tolerance and tumor immunity. Cell Cycle 6, 2478-2485 (2007).

10. Beissert, S. \& Schwarz, T. Ultraviolet-induced immunosuppression: implications for photocarcinogenesis. Cancer Treat. Res. 146, 109-121 (2009).

11. Seite, S., Fourtanier, A., Moyal, D. \& Young, A. R. Photodamage to human skin by suberythemal exposure to solar ultraviolet radiation can be attenuated by sunscreens: a review. Br. J. Dermatol. 163, 903-914 (2010).

12. Meimaridou, E. et al. Mutations in NNT encoding nicotinamide nucleotide transhydrogenase cause familial glucocorticoid deficiency. Nat. Genet. 44, 740-742 (2012).

13. Ji, J. et al. WISP-2 in human gastric cancer and its potential metastatic suppressor role in gastric cancer cells mediated by JNK and PLC-gamma pathways. Br. J. Cancer 113, 921-933 (2015).

14. Choudhury, A. et al. Silencing of ROR1 and FMOD with siRNA results in apoptosis of CLL cells. Br. J. Haematol. 151, 327-335 (2010).

15. Sekiya, M. et al. Absence of Nceh1 augments 25-hydroxycholesterol-induced ER stress and apoptosis in macrophages. J. Lipid Res. 55, 2082-2092 (2014).

16. Latina, A. et al. DeltaNp63 targets cytoglobin to inhibit oxidative stress-induced apoptosis in keratinocytes and lung cancer. Oncogene 35, 1493-1503 (2015).

17. Thu, K. L. et al. SOX15 and other SOX family members are important mediators of tumorigenesis in multiple cancer types. Oncoscience 1, 326-335 (2014).

18. Lin, B. et al. Conversion of $\mathrm{BCl}-2$ from protector to killer by interaction with nuclear orphan receptor Nur77/TR3. Cell 116, 527-540 (2004).

19. Barker, C. E., Ali, S., O'Boyle, G. \& Kirby, J. A. Transplantation and inflammation: implications for the modification of chemokine function. Immunology 143 , 138-145 (2014).

20. Tewari, A., Grys, K., Kollet, J., Sarkany, R. \& Young, A. R. Upregulation of MMP12 and its activity by UVA1 in human skin: potential implications for photoaging J. Invest. Dermatol. 134, 2598-2609 (2014).

21. Kourtidis, A. et al. An RNA interference screen identifies metabolic regulators NR1D1 and PBP as novel survival factors for breast cancer cells with the ERBB2 signature. Cancer Res. 70, 1783-1792 (2010).

22. Suzuki, S. et al. Nur77 as a survival factor in tumor necrosis factor signaling Proc. Natl Acad. Sci. 100, 8276-8280 (2003).

23. Ragusa, S. et al. PROX1 promotes metabolic adaptation and fuels outgrowth of Wnt(high) metastatic colon cancer cells. Cell Rep. 8, 1957-1973 (2014).

24. Chen, J. S., Su, I. J., Leu, Y. W., Young, K. C. \& Sun, H. S. Expression of T-cell lymphoma invasion and metastasis 2 (TIAM2) promotes proliferation and invasion of liver cancer. Int. J. Cancer 130, 1302-1313 (2012).

25. Kumar, N., Wethkamp, N. Waters, L. C. Carr, M. D. \& Klempnauer, K. H. Tumor suppressor protein Pdcd4 interacts with Daxx and modulates the stability of Daxx and the Hipk2-dependent phosphorylation of p53 at serine 46. Oncogenesis 2, e37 (2013).

26. Hansen, M. T. et al. A link between inflammation and metastasis: serum amyloid $\mathrm{A} 1$ and $\mathrm{A} 3$ induce metastasis, and are targets of metastasis-inducing S100A4. Oncogene 34, 424-435 (2015).

27. Thu, K. L. et al. SOX15 is a candidate tumor suppressor in pancreatic cancer with a potential role in Wnt/beta-catenin signaling. Oncogene 33, 279-288 (2014).

28. Hashimoto Y. Effect of Wnt signaling protein (Wisp2/CCN5) on angiogenesis and invasion in prostate cancer. J. Clin. Oncol. 30 (2012) (suppl 5, abstract 227)

29. Chtarbova, S. et al. Murine Nr4a1 and Herpud1 are upregulated by Wnt-1, but the homologous human genes are independent from beta-catenin activation. Biochem. J. 367, 723-728 (2002). Pt 3.

30. Ding, Y., Shen, S., Lino, A. C., Curotto de Lafaille, M. A. \& Lafaille, J. J. Beta-catenin stabilization extends regulatory T-cell survival and induces anergy in nonregulatory T cells. Nat. Med. 14, 162-169 (2008). 
31. Schwarz, T. \& Schwarz, A. DNA repair and cytokine responses. J. Invest. Dermatol. 14, 63-66 (2009).

32. Schwarz, A., Maeda, A., Stander, S., van Steeg, H. \& Schwarz, T. IL-18 reduces ultraviolet radiation-induced DNA damage and thereby affects photoimmunosuppression. J. Immunol. 176, 2896-2901 (2006).

33. Nishigori, C. et al. Evidence that DNA damage triggers interleukin 10 cytokine production in UV-irradiated murine keratinocytes. Proc. Natl Acad. Sci. 93, 10354-10359 (1996).

34. Maeda, A. et al. Enhanced photocarcinogenesis in interleukin-12-deficient mice. Cancer Res. 66, 2962-2969 (2006).

35. Soter, N. A. Acute effects of ultraviolet radiation on the skin. Semin. Dermatol. $\mathbf{9}$ 11-15 (1990).

36. Woodcock, A. \& Magnus, I. A. The sunburn cell in mouse skin: preliminary quantitative studies on its production. Br. J. Dermatol. 95, 459-468 (1976).

37. Bayerl, C., Taake, S., Moll, I. \& Jung, E. G. Characterization of sunburn cells after exposure to ultraviolet light. Photodermatol. Photoimmunol. Photomed. 11, 149-154 (1995).

38. Wolf, P., Cox, P., Yarosh, D. B. \& Kripke, M. L. Sunscreens and T4N5 liposomes differ in their ability to protect against ultraviolet-induced sunburn cell formation, alterations of dendritic epidermal cells, and local suppression of contact hypersensitivity. J. Invest. Dermatol. 104, 287-292 (1995).

39. van Oosten, M. et al. Differential role of transcription-coupled repair in UVBinduced G2 arrest and apoptosis in mouse epidermis. Proc. Natl Acad. Sci. 97, 11268-11273 (2000)

40. Schwarz, A. et al. Interleukin-12 suppresses ultraviolet radiation-induced apoptosis by inducing DNA repair. Nat. Cell Biol. 4, 26-31 (2002).

41. Davies, G. C. et al. Cbl-b interacts with ubiquitinated proteins; differential functions of the UBA domains of c-Cbl and Cbl-b. Oncogene 23, 7104-7115 (2004).
42. Liu, Q., Zhou, H., Langdon, W. Y. \& Zhang, J. E3 ubiquitin ligase Cbl-b in innate and adaptive immunity. Cell Cycle 13, 1875-1884 (2014).

43. Mohapatra, B. et al. An essential role of CBL and CBL-B ubiquitin ligases in mammary stem cell maintenance. Development 144, 1072-1086 (2017).

44. Chitalia, V. et al. c-Cbl, a ubiquitin E3 ligase that targets active beta-catenin: a novel layer of Wnt signaling regulation. J. Biol. Chem. 288, 23505-23517 (2013).

45. Karimaian, A., Majidinia, M., Bannazadeh Baghi, H. \& Yousefi, B. The crosstalk between Wnt/beta-catenin signaling pathway with DNA damage response and oxidative stress: Implications in cancer therapy. DNA Repair 51, 14-19 (2017).

46. Pittayapruek, P., Meephansan, J., Prapapan, O., Komine, M. \& Ohtsuki, M. Role of matrix metalloproteinases in photoaging and photocarcinogenesis. Int. J. Mol. Sci. 17, E868 (2016).

47. $\mathrm{Yu}, \mathrm{J}$. et al. Ubiquitin ligase Cbl-b acts as a negative regulator in discoidin domain receptor 2 signaling via modulation of its stability. FEBS Lett. $\mathbf{5 8 8}$, 1509-1514 (2014)

48. Sha, Y. et al. Modulation of UVB-induced carcinogenesis by activation of alternative DNA repair pathways. Sci. Rep. 8, 705 (2018)

49. Yamazaki, S. et al. Ultraviolet B-induced maturation of CD11b-type langerin(-) dendritic cells controls the expansion of Foxp3 $(+)$ regulatory T cells in the skin. J. Immunol. 200, 119-129 (2018).

50. Singh, T. P., Mayer, G. \& Wolf, P. In vivo siRNA targeting of CD28 reduces UVinduced DNA damage and inflammation. J. Invest. Dermatol. 134, 861-864 (2014).

51. Warde-Farley, D. et al. The GeneMANIA prediction server: biological network integration for gene prioritization and predicting gene function. Nucleic Acids Res. 38, W214-W220 (2010). Web Server issue. 